Artículo

\title{
Métodos de Griffing: revisión sobre su importancia y aplicación en fitomejoramiento convencional
}

\author{
Claudia Saavedra Guevara ${ }^{1}$ \\ Delfina de Jesús Pérez López ${ }^{2 \S}$ \\ Andrés González Huerta² \\ J. Ramón Pascual Franco Martínez ${ }^{2}$ \\ Martin Rubí Arriaga ${ }^{2}$ \\ José Francisco Ramírez Dávila² \\ ${ }^{1}$ Programa de Doctorado en Ciencias Agropecuarias y Recursos Naturales-Universidad Autónoma del \\ Estado de México-Instituto de Ciencias Agropecuarias y Rurales-Campus Universitario 'El Cerrillo'. \\ Toluca, Estado de México, México. Tel. 722 2965552, ext. 117. (pcarn@uaemex.mx). ${ }^{2}$ Centro de \\ Investigación y Estudios Avanzados en Fitomejoramiento-Facultad de Ciencias Agrícolas. Toluca, Estado \\ de México, México. AP. 435. Tel. 722 2965518, ext. 148. (csaavedrag001@alumno.uaemex.mx; \\ agonzalezh@uaemex.mx; jrfrancom@uaemex.mx; mrubia@uaemex.mx; jframirezd@uaemex.mx).
}

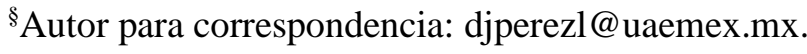

\section{Resumen}

La estimación de parámetros genéticos en plantas y animales es de gran relevancia en las ciencias agropecuarias y biológicas. En este contexto, los efectos y varianzas de aptitud combinatoria, la heredabilidad, la heterosis, la respuesta a la selección, la identificación de progenitores y cruzas simples sobresalientes, así como la predicción de híbridos de mayor producción y calidad, depende del tipo de diseño de apareamiento y experimental elegido. En este estudio se analizan las cuatro metodologías de Griffing con relación a las de Hayman y Jinks y Gardner y Eberhart; se subrayan las suposiciones consideradas en éstas y se comentan las similitudes que existen entre ellas. Se incluyen los ocho modelos matemáticos que Griffing discutió y que frecuentemente se utilizan para aplicar algún paquete estadístico, se citan algunas investigaciones realizadas en la última década, y se sugieren algunos softwares para su análisis genético-estadístico.

Palabras clave: cruzas dialélicas, modelos y paquetes genético-estadísticos, parámetros genéticos.

Recibido: marzo de 2021

Aceptado: junio de 2021 


\section{Introducción}

Los experimentos dialélicos están relacionados con estudios genéticos, así como con programas de fitomejoramiento, producción de semillas, generación, validación, aplicación y transferencia de tecnología (Martínez, 1988; González et al., 2007a, b; Pérez et al., 2019; González et al., 2019). Los diseños genéticos que se usan para formar familias de autohermanos, medios hermanos y hermanos completos, dentro y entre poblaciones de la misma variedad, raza, especie y género, son cruzas entre dos progenitores, policruza, cruza de prueba, diseños I, II y III de Carolina del norte, métodos 1, 2, 3 y 4 de Griffing, línea por probador (Shattuck et al., 1993; Harriman y Nwammadu, 2016; Nduwumuremyi et al., 2013; Awata et al., 2018), triple cruza de prueba, análisis tri y cuatrialélico, retrocruza y dialélicos incompletos (Kempthorne y Curnow, 1961; Soriano, 2000; Mumtaz et al., 2015).

Otro análisis propuesto en especies autógamas o en las que se pueden derivar fácilmente líneas puras, se atribuye a Jinks y Hayman (1953); Hayman (1954 a, b); Jinks (1954), fundamentado en la estimación de seis tipos de varianzas y covarianzas entre parientes. Gardner y Eberhart (1966) diseñaron otra metodología en la que cada progenitor es una población con apareamiento aleatorio, está en equilibrio Hardy-Weinber, y hay dos alelos por locus. En la evaluación del material genético en áreas homogéneas se ha aplicado el diseño completamente al azar. Con un gradiente de variación no deseable, pero predecible, se ha elegido bloques completos al azar.

Con dos gradientes de heterogeneidad ambiental, uno perpendicular al otro, se ha empleado un cuadrado latino o algún látice (Gomez y Gomez, 1984; Martínez, 1988; Borojevic, 1990), pero han sido más usadas las series de experimentos en tiempo y espacio en bloques completos al azar o en látices (González et al., 2007a, b; Martínez, 1988; Moore y Dixon, 2015; González et al., 2019). Los diseños de apareamiento y experimentales se usan para estimar efectos y varianzas genéticas y ambientales, heredabilidad, heterosis, respuesta a la selección, predicción de híbridos, como material para nuevos programas de fitomejoramiento o como sugerencia para siembra comercial (Rodríguez et al., 2016; Vesali et al., 2020; Aditika et al., 2020; Vasconcelos et al., 2020). En el contexto anterior, se hará énfasis en los cuatro métodos de Griffing (1956a, b), que son los más utilizado desde los 1950's. También se citan algunos paquetes estadísticos disponibles en sus sitios WEB, como versiones académicas o comerciales.

\section{Materiales y métodos}

\section{Suposiciones usadas en una cruza dialélica}

Christie y Shattuck (1992) discutieron cuatro diseños básicos considerando la ACG y ACE (Griffing, 1956b), los arreglos de varianzas y covarianzas (Jinks y Hayman, 1953; Hayman, 1954b; Jinks, 1954; Jinks, 1956), los efectos aditivos y dominantes (Gardner y Eberhart, 1966; Eberhart y Gardner, 1966) y los dialélicos incompletos (Kempthorne y Curnow, 1961). En éstos, como en otras técnicas dialélicas, hay alguna suposición o restricción para la estimación de parámetros genético-estadísticos (Martínez, 1988; Sahagún, 1998; Soriano, 2000; Awata et al., 2018).

Los cuatro métodos de Griffing no están condicionados por hipótesis o suposiciones genéticas (Wright, 1985; Christie y Shattuck, 1992; Shattuck et al., 1993; Hallauer et al., 2010). En la técnica de Hayman y Jinks se considera segregación diploide, progenitores homocigóticos, no diferencias 
entre cruzas directas y recíprocas, ausencia de epistasis y no ligamiento entre genes, ausencia de alelos múltiples, y distribución independiente de los genes en los progenitores. En las metodologías de Gardner y Eberhart sólo se asume que cada progenitor se encuentra en equilibrio HardyWeimberg y que en cada locus hay solo dos alelos.

\section{Métodos de Griffing}

Griffing (1956 b) propuso una técnica dialélica para estimar ACG y ACE, así como la naturaleza y el tipo de acción génica involucrada en la herencia de caracteres cuantitativos que se manifiestan en plantas y animales; ésta puede variar en su diseño y en su análisis biométrico si progenitores, cruzas simples directas o cruzas simples recíprocas (p, CD y CR, respectivamente) son incluidos. Con base en las combinaciones que se pueden formar cuando se consideran p padres, en parejas diferentes $\left(\mathrm{C}_{2}^{\mathrm{p}}\right)$, se tendrá: método 1: las $\mathrm{p}^{2}$ familias se denominan dialélico completo e incluyen $\mathrm{p}$, $\mathrm{CD}$ y $\mathrm{CR}\left(\mathrm{p}+\mathrm{C}_{2}^{\mathrm{p}}+\mathrm{C}_{2}^{\mathrm{p}}\right)$. Método 2: en este dialélico medio hay $\mathrm{p}+\mathrm{C}_{2}^{\mathrm{p}}=\mathrm{p}(\mathrm{p}+1) / 2$ familias ( $\mathrm{p}$ y $\left.C D\right)$. Método 3: este dialélico modificado analiza $C D$ y $C R$; hay $C_{2}^{p}+C_{2}^{p}=p^{2}-p$ familias. Método 4: se obtienen $\mathrm{C}_{2}^{\mathrm{p}}=\mathrm{p}(\mathrm{p}-1) / 2$ familias (sólo $\mathrm{CD}$ ).

\section{Modelos genético-estadísticos}

En los ocho análisis que se originan combinando los modelos de efectos fijos (I) y aleatorios (II) con sus cuatro métodos, Griffing (1956b) centra su discusión en un diseño de bloques completos al azar, para un ambiente, hay b repeticiones, a genotipos y c observaciones en cada parcela experimental.

Método 1

Modelo I: $x_{i j}=\mu+g_{i}+g_{j}+s_{i j}+r_{i j}+\frac{1}{b c} \sum_{k} \sum_{l} e_{i j k l}\left\{\begin{array}{l}i, j=1, \ldots, p \\ k=1, \ldots, b \\ 1=1, \ldots, c\end{array}\right\}$

Donde: $\mu=$ media poblacional; $\mathrm{g}_{\mathrm{i}}, \mathrm{g}_{\mathrm{j}}=$ efectos de ACG para los progenitores $\mathrm{i}, \mathrm{j}, \mathrm{s}_{\mathrm{ij}}=$ efectos de ACE para esa pareja de progenitores; $\mathrm{r}_{\mathrm{ij}}=$ efecto originado por su cruza recíproca; $\mathrm{e}_{\mathrm{ijk} l}=$ efecto ambiental que afecta a cada ijkl- ésima observación, ésta última también se conoce como residual del modelo. Las componentes de los modelos I, que se muestran a continuación, se definen en los mismos términos que para el método 1 .

Modelo II: $\mathrm{x}_{\mathrm{ij}}=\mu+\mathrm{g}_{\mathrm{i}}+\mathrm{g}_{\mathrm{j}}+\mathrm{s}_{\mathrm{ij}}+\mathrm{r}_{\mathrm{ij}}+\frac{1}{\mathrm{~b}} \sum_{\mathrm{k}} \mathrm{b}_{\mathrm{k}}+\frac{1}{\mathrm{~b}} \sum_{\mathrm{k}}(\mathrm{bv})_{\mathrm{ijk}}+\frac{1}{\mathrm{bc}} \sum_{\mathrm{k}} \sum_{\mathrm{l}} \mathrm{e}_{\mathrm{ijk} \mathrm{k}}$. En los cuatro métodos, con excepción de $\mu$, las otras componentes son variables aleatorias.

Método 2

Modelo I: $x_{i j}=\mu+g_{i}+g_{j}+s_{i j}+\frac{1}{b c} \sum_{k}\left\{\begin{array}{c}i, j=1, \ldots, p \\ k=1, \ldots, b \\ 1=1, \ldots, c\end{array}\right\}$

Modelo II: $\mathrm{x}_{\mathrm{ij}}=\mu+\mathrm{g}_{\mathrm{i}}+\mathrm{g}_{\mathrm{j}}+\mathrm{s}_{\mathrm{ij}}+\frac{1}{\mathrm{~b}} \sum_{\mathrm{k}} \mathrm{b}_{\mathrm{k}}+\frac{1}{\mathrm{~b}} \sum_{\mathrm{k}}(\mathrm{bv})_{\mathrm{ijk}}+\frac{1}{\mathrm{bc}} \sum_{\mathrm{k}} \sum_{\mathrm{l}} \mathrm{e}_{\mathrm{ijkl}}$ 
Método 3

Modelo I: $x_{i j}=\mu+g_{i}+g_{j}+s_{i j}+r_{i j}+\frac{1}{b c} \sum_{k} \sum_{1} e_{i j k l}\left\{\begin{array}{c}i, j=1, \ldots, p \\ k=1, \ldots, b \\ 1=1, \ldots, c\end{array}\right\}$

Modelo II: $x_{i j}=\mu+g_{i}+g_{j}+s_{i j}+r_{i j}+\frac{1}{b} \sum_{k} b_{k}+\frac{1}{b} \sum_{k}(b v)_{i j k}+\frac{1}{b c} \sum_{k} \sum_{1} e_{i j k l}$

Método 4

Modelo $\mathrm{I}: \mathrm{x}_{\mathrm{ij}}=\mu+\mathrm{g}_{\mathrm{i}}+\mathrm{g}_{\mathrm{j}}+\mathrm{s}_{\mathrm{ij}}+\frac{1}{\mathrm{bc}} \sum_{\mathrm{k}} \sum_{\mathrm{l}} \mathrm{e}_{\mathrm{ijkl}}\left\{\begin{array}{c}\mathrm{i}, \mathrm{j}=1, \ldots, \mathrm{p} \\ \mathrm{k}=1, \ldots, \mathrm{b} \\ 1=1, \ldots, \mathrm{c}\end{array}\right\}$

Modelo II: $\mathrm{x}_{\mathrm{ij}}=\mu+\mathrm{g}_{\mathrm{i}}+\mathrm{g}_{\mathrm{j}}+\mathrm{s}_{\mathrm{ij}}+\frac{1}{\mathrm{~b}} \sum_{\mathrm{k}} \mathrm{b}_{\mathrm{k}}+\frac{1}{\mathrm{~b}} \sum_{\mathrm{k}}(\mathrm{bv})_{\mathrm{ijk}}+\frac{1}{\mathrm{bc}} \sum_{\mathrm{k}} \sum_{\mathrm{l}} \mathrm{e}_{\mathrm{ijkl}}$

\section{Aplicaciones en las cuatro metodologías}

El método 1 se ha usado más frecuentemente en fitomejoramiento para estimar efectos y varianzas de aptitud combinatoria, efectos recíprocos y maternos, acción génica, heterosis, heredabilidad, predicción de híbridos sobresalientes y respuesta a la selección, como puede constatarse en los estudios realizados por González et al. (2007 b); Amissah et al. (2019); Lima et al. (2019); Yaw et al. (2020), entre otros. La estimación de parámetros genéticos con el método 2 en papa (Solanum tuberosum L.); maíz (Zea mays L.); gerbera (Gerbera x hybrida), entre otras especies, han sido realizados por Muhiuyuza et al. (2016); Oliveira et al. (2016); Mbusa et al. (2017); Rivera-Colín et al. (2019); Muhumuza et al. (2020). Éste se ha usado para estimar aptitud combinatoria, heterosis, heredabilidad, acción génica, depresión endogámica y predicción de híbridos superiores.

Con el método 3, Rodríguez-Pérez et al. (2016); Ngalio et al. (2019); Barreta et al. (2019); Vesali et al. (2020) han estimado parámetros genéticos en maíz, camote (Ipomoea batatas L.) y papa, pero en éstos no se estimó heterosis debido a que en el análisis no se incluyen progenitores. En los estudios de Muhinyuza et al. (2016); Anyanga et al. (2016); Barroso et al. (2019); Aditika et al. (2020); Vasconcelos et al. (2020) se ha destacado la relevancia que tiene el método 4 en la estimación de parámetros genéticos, en éstos se ha dado prioridad al análisis y discusión de los efectos de aptitud combinatoria general y específica, heredabilidad en sentido amplio y estrecho, y en la predicción de híbridos de mayor producción.

\section{Software disponible}

Algunos paquetes estadísticos que se encuentran disponibles en la internet, para el análisis de experimentos de cruzas dialélicas, son: Statistical Analysis System (SAS), Agrobase, Indostat, AGD-R (Analysis of Genetic Design in R), PBTools (Plant Breeding Tools in R), TNAUSTAT (Plant Breeding-Heterosis), OPStat, y Genes, entre otros. 


\section{Resultados y discusiones}

El fitomejoramiento se puede dividir en tres etapas: la reunión o creación de un acervo de germoplasma variable, la selección de individuos superiores en el acervo y su utilización para crear una nueva y mejor variedad. En éstas la estimación de parámetros genéticos, como la varianza genética y la heredabilidad, pueden ser de gran valor (Dudley y Moll, 1969). En estudios genéticos y de fitomejoramiento el diseño de apareamiento y ambiental (experimental), así como el arreglo de siembra que se elija son muy importantes para generar el tipo de familia requerido, para disminuir el residual del modelo matemático, para optimizar el tiempo destinado al ensayo, y para estimar con mayor precisión los parámetros poblacionales que permitan obtener conclusiones correctas (Sahagún, 1998; Mumtaz et al., 2015; Harriman y Nwammadu, 2016; Awata et al., 2018).

Nduwumuremyi et al. (2013) puntualizaron que la elección de un grupo de progenitores y de un diseño genético apropiados son elementos clave para desarrollar programas de fitomejoramiento exitosos y que éstos, están condicionados por los objetivos del estudio, el tiempo, el espacio, los costos y otras restricciones biológicas. Con relación al diseño de apareamiento deberá considerarse el tipo de polinización predominante (por viento o insectos), la forma de diseminación del polen (por viento o insectos), si hay esterilidad genética o citoplásmica, los objetivos de la investigación, y el tamaño de la población requerida, entre otros (Nduwumeremyi et al., 2013; Mumtaz et al., 2013; Fasahat et al., 2016).

Desde los 1950's se han diseñado diversas técnicas dialélicas pero las que más se usan son las de Griffing, Hayman y Jinks, y Gardner y Eberhart (Christie y Shattuck, 1992; Shattuck et al., 1993; Hallauer et al., 2010; Nduwumuremyi et al., 2013). Cockerham (1963) clasificó los diseños de apareamiento en categorías de uno, dos, tres y cuatro factores, dependiendo del número de ancestros por progenie sobre los cuales se ejerce control. La policruza (un factor) es suficiente para detectar variabilidad genética; con la cruza dialélica, con los diseños I, II y III de Carolina del norte y con el dialélico parcial (dos factores) se puede estimar varianza aditiva y de dominancia, la epistasis o el ligamiento entre genes se puede calcular usando cruzas tri y cuatrialélicas (tres y cuatro factores) o con la combinación de alguno de estos diseños.

Fasahat et al. (2016) comentaron que los diseños genéticos que más frecuentemente se han usado en las últimas décadas son la cruza dialélica, el diseño factorial de Carolina del Norte (método II) y la cruza línea x probador (mestizos). En éstos se han discutido los resultados con base en la estimación de los efectos y varianzas de aptitud combinatoria general y específica en cultivos como trigo, cártamo (Carthamus tinctorius L.), arroz (Oriza sativa L.), sorgo (Sorghum bicolor (L) Moench), alfalfa (Medicago sativa L.), chile (Capsicum annum L.), chícharo de vaca (Vigna unguiculata L.), jitomate (Solanum lycopersicum L.), ajonjolí (Sesamum indicum L.) y linaza (Linum usitatissimum L.), entre otros.

La evaluación de las progenies de una cruza dialélica en varios años y localidades usando un diseño y un análisis genético-estadístico apropiado podría ser compleja, pero esencial, para determinar su estructura genética, ambiental y de interacción genotipo-ambiente, su contribución en la estimación de los efectos y varianzas de aptitud combinatoria, heterosis, heredabilidad, respuesta a la selección y predicción de híbridos sobresalientes (Sahagún, 1990; González et al., 2007a, b; Harriman y Nwammadu, 2016; Fasahat et al., 2016; Awata et al., 2018). 
Los componentes de varianza genética estimada por medio de los diseños de apareamiento se pueden igualar con las covarianzas entre parientes. Las únicas suposiciones necesarias son que los progenitores sean miembros aleatorios de la población genética y que los errores experimentales sean independientes. Las correlaciones ambientales se evitan aleatorizando las progenies; una varianza negativa puede atribuirse a una desviación causada por el tipo de muestreo y por el tamaño de la muestra (Dudley y Moll, 1969).

La técnica del análisis de varianza (Anava) se usa para dividir la variabilidad estimada en uno o más experimentos en diferentes componentes según el modelo genético-estadístico aplicado a variables cuantitativas. También se emplea en rasgos cualitativos después de transformar los datos originales para que éstos cumplan con las suposiciones de que los efectos genotípicos y ambientales sean aditivos, y que los errores experimentales o residuales de los modelos, se distribuyan normal e independientemente con una varianza común (Sahagún, 1990; Sahagún, 1998). Si las diferencias entre genotipos son significativas $(p \leq 0.05)$ se realiza el análisis dialélico, con alguna de las metodologías consideradas en este o en otros estudios (Griffing, 1956b; Shattuck et al., 1993; González et al., 2007a, b; Pérez et al., 2020).

González et al. (2007b); Pérez et al. (2020) dividieron la variabilidad de un dialélico completo formado con líneas endogámicas de maíz en sumas de cuadrados parciales y contrastes mutuamente ortogonales para probar las hipótesis sobre progenitores $(\mathrm{P})$, cruzas directas $(\mathrm{CD})$, cruzas recíprocas (CR), $\mathrm{P}$ versus $\mathrm{CD}$ y $\mathrm{CD}$ versus $\mathrm{CR}$, cuando sus efectos fueron significativos se aplicó el método 1 de Griffing (1956b), como lo sugirieron Shattuck et al. (1993). El primer contraste calcula heterosis promedio, también estimable en el Anava con el método de Gardner y Eberhart (1966), pero con ninguno de Griffing (1956b) y el segundo contraste calcula indirectamente efectos maternos y recíprocos, también estimables con el Anava con los métodos 1 y 3 de Griffing (1956b).

Singh (1973a, b) desarrolló los procedimientos estadísticos para el análisis de datos de una cruza dialélica en varios ambientes, pero Zhang y Kang (1997); Zhang et al. (2005) mostraron como estimar efectos recíprocos usando SAS con énfasis en su partición en efectos maternos y no maternos en series de experimentos, como lo sugerió Cockerham (1963). Ellos comentaron que este tipo de partición permite saber si los efectos maternos o los factores extranucleares están involucrados en la expresión de un rasgo fenotípico.

Kearsey (1965) derivó familias de la misma población usando cruzas entre dos progenitores, diseños I y II de Carolina del norte, dialélico medio de Hayman (1954) y dialélico parcial de Kempthorne y Curnow (1961). La única restricción fue que para la estimación de los componentes de varianza el número de cruzas en cada metodología, incluyendo las autofecundaciones, fuera igual o cercano a 45. Se concluyó que el método de Hayman y Jinks (1954) proporciona más información que cualquier otro diseño, pero requiere que se cumplan un mayor número de suposiciones genéticas. Baker (1978) agrupó los métodos II y III de Gardner y Eberhart (1966), 2 y 4 de Griffing (1956b) y el de Hayman (1954) para discutir los problemas que surgen en un análisis dialélico, desde el punto de vista estadístico existen controversias críticas sobre la elección de un modelo de efectos genotípicos fijo y aleatorio y desde el punto de vista genético, las suposiciones sobre la distribución independiente de los genes en los progenitores es difícil de satisfacer y la hipótesis de ausencia de epistasis y de ligamiento de genes también puede ser incorrecta.

Los métodos de Griffing (1956b) se han preferido sobre los de Hayman y Jinks y los de Gardner y Eberhart (1966) debido a que no están condicionados por suposiciones genéticas. Además, el análisis, si no se dispone de software especializado, es más complejo en las dos últimas. Los 
métodos de Griffing (1956b) y de Gardner y Eberhart (1966) pueden aplicarse en especies autógamas y alógamas, incluso poliploides como la papa (Solanum tuberosum L.) y los de Hayman y Jinks (1954) están restringidos a progenitores homocigóticos o endogámicos (Baker, 1978; Christie y Shattuck, 1992; Awata et al., 2018). Christie y Shattuck (1992) comentaron que los análisis de Gardner y Eberhart (1966) son similares a los de Hayman (1954a, b) y a uno de Griffing (1956 b). En Zhang y Kang (1997) se analizan otras similitudes y diferencias que existen entre los cuatro métodos de Griffing.

La principal limitante que muestran las metodologías de Griffing (1956a, b) tiene que ver con el número de progenitores elegido: con cuatro padres hay tres grados de libertad que no permiten estimar con confiabilidad la hipótesis para ACG y con 20 de ellos habrá mayor precisión en su estimación, pero se generará un ensayo inmanejable (400 cruzamientos) si se elige el método 1, independientemente de la aplicación del modelo I o II (González et al., 2007a, b; Awata et al., 2018; González et al., 2020; Pérez et al., 2020). Christie y Shattuck (1992) comentaron que en la mayoría de los experimentos dialélicos que revisaron se incluyeron entre 6 y 10 progenitores.

Utilizar un mayor número de padres permitirá obtener información más confiable, pero su número óptimo deberá ser tal que permita justificar la información adicional que se obtenga y es necesario definir con anticipación a la población de referencia. Cuando los progenitores fueron considerados como una muestra de una población ancestral fueron sugeridos entre 17 y 19 padres o entre tres y cinco ensayos con ocho de ellos cada uno (Hayward, 1979). En las investigaciones que fueron consideradas en el presente estudio se observó que el número de progenitores para los métodos 1 , 2, 3 y 4 de Griffing (1956b) varió de 4 a 12, 5 a 12, 7 a 13, y 4 a 16, respectivamente. Fasahat et al. (2016) reportaron que para el dialélico el número de progenitores ensayado varió de 4 a 15 y para el dialélico medio osciló de 5 a 12.

Griffing (1956b) discutió ocho análisis que se originan al combinar sus cuatro métodos con las dos suposiciones que se hacen con relación a la naturaleza de muestreo del material experimental (modelos I y II). Shattuck et al. (1993) destacaron que son 16 análisis posibles, si además de lo anterior, se incluyen los modelos mixtos A y B. En la realidad habría más combinaciones si se consideran las series de experimentos en tiempo y espacio o los arreglos de parcelas divididas ensayados bajo los diseños experimentales completamente al azar, bloques completos al azar o algún látice (Gomez y Gomez, 1984; Sahagún, 1998; Moore y Dixon, 2014; Pérez et al., 2019).

La elección de alguna de las metodologías de Griffing (1956b) depende del tipo de material experimental considerado y de los objetivos de la investigación. Cuando sólo son del interés las cruzas $\mathrm{F}_{1}$ es más apropiada la aplicación de los métodos 3 y 4 para estimar los efectos (modelo I) o las varianzas (modelo II) de ACG y ACE. Si hay duda con relación a la presencia de genes ligados al sexo o de efectos maternos el método 3 es el más adecuado. Si el objetivo principal es identificar líneas sobresalientes que serán utilizadas en la formación de una variedad sintética los progenitores deberán incluirse en el experimento y se emplearán los métodos 1 o 2 para su análisis genéticoestadístico (Griffing, 1956b; Baker, 1978; Christie y Shattuck, 1992; Shattuck et al., 1993; González et al., 2007a, b).

La ACG está relacionada con efectos génicos aditivos y la ACE con dominancia o epistasis (Sprague y Tatum, 1942; Dudley y Moll, 1969; Baker, 1978; Christie y Shattuck, 1992; Awata et $a l ., 2018)$. En las diferentes técnicas de selección son más importantes los efectos de aditividad y 
en los programas por hibridación son esenciales los efectos no aditivos (Borojevic, 1990; Mumtaz et al., 2015; Harriman y Nwammadu, 2016; Awata et al., 2018). Con relación a la predicción de híbridos $F_{1}$, Dudley y Moll (1969) puntualizaron que sólo se justifica cuando la proporción de la varianza de dominancia sobre la varianza aditiva se encuentre en el rango de sobredominancia o cuando se obtengan estimaciones de la varianza epistática que incluya varianza de dominancia. Ellos no recomiendan el uso de algún tipo de diseño en látice debido que éstos se confunden con las medias de diferencias genéticas y ambientales, por lo que éstos no son satisfactorios.

En especies de polinización libre (plantas alógamas), cuando la autofecundación origina depresión endogámica que causa alta mortandad de plantas, como sucede frecuentemente en la alfalfa (Medicago sativa L.), los estudios de aptitud combinatoria general y específica podrían estar restringidos al uso de progenitores no endogámicos o parcialmente endogámicos, con una sola generación de autofecundación; si F, el coeficiente de endogamia, es igual o menor a 0.75, la cruza dialélica no es recomendable. En esta última situación y cuando el objetivo sea estimar efectos de ACG, los métodos de policruza, línea x probador y dialélico parcial podrían ser los más apropiados (Griffing, 1956b; Christie y Shattuck, 1992; Shattuck et al., 1993; González et al., 2007a).

En tomate de cáscara (Physalis ixocarpa Brot. ex Horm.) existe incompatibilidad gametofítica causada por dos genes independientes con alelos múltiples que impiden la formación de líneas altamente endogámicas e híbridos usando técnicas de fitomejoramiento convencionales (Mulato et $a l ., 2020)$ por lo que, con esta restricción biológica, sería más recomendable aplicar alguna técnica de selección (Peña y Márquez, 1990), practicar hibridación intervarietal con familias de medios hermanos maternos (Peña et al., 1999) o realizar cruzas planta a planta (Santiaguillo et al., 2004).

Ningún método de Griffing (1956b) considera epistasis y ligamiento; para su estimación debe usarse uno de éstos junto con la metodología de Hayman (1954) o si el objetivo fuera también estimar heterosis, ambas técnicas junto con la de Gardner y Eberhart (1966), aun cuando incrementarían los costos y el tiempo destinado a la evaluación de un grupo de cruzas dialélicas, serían las más deseables para realizar una evaluación integral (Hallauer et al., 2010; Muntaz et al., 2015; Awata et al., 2018; Pérez et al., 2020).

Para SAS se han diseñado los códigos y programas para las metodologías de Griffing (1956 b), Hayman (1954) y Gardner y Eberhart (1966), entre otros, para analizar datos con los diseños experimentales completamente al azar, bloques completos al azar y algún látice en uno o más factores (Martínez, 1988; Kang y Zhang, 1977; Zhang et al., 2005; Makumbi et al. (2018), si no se dispone de recursos para adquirir una licencia comercial, podría descargarse una versión académica de prueba, con vigencia hasta por un año.

AGD-R, PBTools y Genes también son buenas opciones; éstas se pueden descargar gratuitamente de los portales electrónicos del Centro Internacional de Mejoramiento de Maíz y Trigo (CIMMYT; Rodríguez et al., 2018), del Instituto Internacional de Investigación en Arroz (IRRI, 2014) y de la Universidad Federal de Vicosa (Brasil; Cruz, 2013), respectivamente. Agrobase e Indostat sólo pueden utilizarse con licencia comercial, pero ambos son más amigables que los softwares anteriores. Tnaustat y OPStat, disponibles gratuitamente en sus portales electrónicos, sólo analizan experimentos para un ambiente (año o localidad). En Mastache et al. (1999 a, b); Mastache y Martínez (2003); Sestras et al. (2018); González et al. (2020) se consideran códigos, programas y algoritmos para el análisis de Griffing con SAS u otro software. 


\section{Conclusiones}

La elección de progenitores adecuados y de un buen diseño de apareamiento y experimental son las claves para lograr un esquema exitoso de mejoramiento. Desde un punto de vista estadístico la parte critica del uso de los diseños dialélicos sería la elección de un modelo de efectos fijos o aleatorios, desde el punto de vista genético, para interpretar los resultados, la suposición de la distribución independiente de los genes en los progenitores no es muy aceptable en la práctica, el segundo supuesto, la ausencia de epistasis y de ligamiento entre genes, es a veces también incorrecto. La epistasis afecta la estimación de las varianzas de ACG y ACE. Como alternativa a la interpretación genética de los resultados, la descripción estadística del análisis dialélico puede ser usada para contestar preguntas concernientes a la importancia de la ACE y la predictibilidad del comportamiento de los híbridos usando ACG o el comportamiento de los progenitores.

\section{Literatura citada}

Aditika-Kanwar, H. S.; Priyanka-Shalini, S. and Saurabh, S. 2020. Heterotic potential, potence ratio, combining ability and genetic control of quality and tield traits in bell pepper under net-house conditions of NW himalayas. Agric. Res. 9(4):526-535.

Amissah, S.; Osekre, E. A.; Nyadanu, D.; Akromah, R.; Afun, J. V. K.; Amoah, R. A.; Owusu, G. A. and Adejumobi, I. I. 2019. Inheritance and combining ability studies on grain yield and resistance to maize weevil (Sitophilus zeamais, mostchulsky) among extra early quality protein maize inbred lines. Ecol. Genet. Gen. 12(1):100043.

Anyanga, W. O.; Rubalhayo, P.; Gibson, P. and Okori, P. 2016. Combining ability and gene action in sesame (Sesamum indicum L.) elite genotypes by diallel mating design. J. Plant Breed. Crop Sci. 8(11):250-256.

Awata, L. A. O.; Tongoona, P.; Danquah, E.; Efie, B. E. and Marchelo-Dragga, P. W. 2018. Common mating designs in agricultural research and their reliability in estimation of genetic parameters. IOSR. J. Agric. Vet. Sci. 11(7):16-36.

Baker, R. J. 1978. Issues in diallel analysis. Crop Sci. 18(4):533-536.

Barreta, D.; Nardino, M.; Konflanz, V. A.; De-Pelegrin, A. J.; Szareski, V. J.; Carvelho, I. R; DeSouza, V. Q.; De-Oliveira, A. C. and Da-Maia, L. C. 2019. Diallelic analysis of endogamic maize lines with emphasis on agronomic trais of tassel in different environments. J. CropSci. Biotech. 22(2):101-111.

Borojevic, S. 1990. Principles and methods of plant breeding. Developments in crop science 17. Elservier science publishing company inc. Amsterdam, The Netherlands. 368 p.

Barroso, C. J. T.; Dos-Santos, P. R.; Daher, R.; Francesconi, S. W.; Kesia, V. A.; Rodrigues, N. M. Da-Silva, C. D.; Vivas, M.; Teixeira, D. A. J. A. and Menezes, D. 2019. Estimation of genetic merit of diallel hybrids of seet pepper by mixed models. Ciencia Rural, Santa Maria. 48(08):1-7).

Christie, B. R. and Shattuck, V. I. 1992. The diallel cross: design, analysis and use for plant breeders. Plant Breed. Rewiews. 9(1):9-36.

Cockerham, C. C. 1963. Estimation of genetic variances. In: Hanson, W. D. and Robinson, H. F. (Ed.). Statistical genetics and plant breeding. NRC Publ. 982. Natl. Acad. Sci. Natl. Res. Council. Washington, DC. 53-93 pp.

Cruz, C. D. 2013. Genes-a software package for analysis in experimental statistics and quantitative genetics. Acta scientiarum. Agronomy. 35(3):271-276. 
Dudley, J. W. and Moll, R. H. 1969. Interpretation and use of estimates of heritability and genetic variance in plant breeding. Crop Sci. 9(3):257-262.

Eberhart, S. A. and Gardner, C. O. 1966. A general model for genetic effects. Biometrics. 22(4):864-881.

Fasahat, P.; Rajabi, A.; Rad, J. M. and Derera, J. 2016. Principles and utilization of combining ability in plant breeding. Biom. Bio. Inter. J. 4(1):1-22.

Gardner, C. O. and Eberhart, S. A. 1966. Analysis and interpretation of the variety cross diallel and related populations. Biometrics. 22(3):439-452.

Griffing, B. 1956a. A generalized treatment of diallel cross in quantitative inheritance. Heredity. 10(1):31-50.

Griffing, B. 1956b. Concept of general and specific combining ability in relation to diallel crossing systems. Aust. J. Biol. Sci. 9(4):463-493.

Gomez, K. A. and Gomez, A. A. 1984. Statistical procedures for agricultural research. $2^{\text {nd }}$ (Ed.). John Wiley and Sons, Inc. Singapore. 690 p.

González, H. A.; Sahagún, C. J. y Pérez, L. D. J. 2007a. Estudio de ocho líneas de maíz en un experimento dialélico incompleto. Rev. Cienc. Agríc. Inf. 16(1):3-9.

González, H. A.; Pérez, L. D. J.; Sahagún, C. J.; Norman, M. T.; Balbuena, M. A. y Gutiérrez, R. F. 2007b. Análisis de una cruza dialélica completa de líneas endogámicas de maíz. Rev. Cienc. Agríc. Inf. 16(1):10-17.

González, H. A.; Pérez, L. D. J.; Rubí, A. M.; Gutiérrez, R. F.; Franco, M. J. R. P. y Padilla, L. A. 2019. InfoStat, InfoGen y SAS para contrastes mutuamente ortogonales en experimentos en bloques completos al azar en parcelas subdivididas. Rev. Mexic. Cienc. Agríc. 10(6):1417-1431.

Hallauer, A. R.; Carena, M. J. and Filho, J. B. M. 2010. Quantitative genetics in maize breeding. $6^{\text {th }}$ (Ed.). Springer, Iowa, USA. 663 p.

Harriman, J. C. and Nwammadu, C. A. 2016. Utilization of diallel analyses for heritability, GCA and SCA studies in crop improvement. Am. Adv. J. Biol. Sci. 2(5):159-167.

Hayman, B. I. 1954 a. The analysis of variance of diallel tables. Biometrics. 10(2):235-244.

Hayman, B. I. 1954 b. The theory and analysis of diallel crosses. Genetics. 39(6):789-809.

Hayward, M., D. 1979. The application of the diallel cross to outbreeding crop species. Euphytica. 28(3):729-737.

IRRI. 2014. International rice research institute. Plant breeding tools (pbtools). User's manual, version 1.4. Biometrics and breeding informatics. Plant Breed. Gen. Biotechnol. Division. Irri. 198 p.

Jinks, J. L. and Hayman, B. I. 1953. The analysis of diallel cross. Maize genetics cooperation newsletter. 27(1):48-54.

Jinks, J. L. 1954. The analysis of continuous variation in a diallel cross in nicotiana rustic varieties. Genetics. 39(1):767-788.

Kearsey, M. J. 1965. Biometrical analysis of a random mating population: a comparison of five experimental designs. Heredity. 20(2):205-235.

Kemthorne, O. and Curnow, R. N. 1961. The partial diallel cross. Biometrics. 17(2):229-250.

Lima, D. N. T.; De-França, S. F.; Souza, D. R. C. and Ferreira, D. S. E. 2019. Watermelon general and specific combining ability. Communicate Scientiae. 10(1):132-140.

Makumbi, D.; Alvarado, G.; Crossa, J. and Burgueño, J. 2018. Sashaydiall: a sas program for hayman's diallel analisis. Crop Sci. 58(4):1605-1615.

Martínez, G. A. 1988. Diseños Experimentales: métodos y elementos de teoría. (Ed.). Trillas, México, DF. 756 p. 
Mastache, L. A. A.; Martínez, G. A. y Castillo, M. A. 1999a. Los mejores predictores lineales e insesgados (MPLI) en los diseños dos y cuatro de griffing. Agrociencia. 33(1):81-91.

Mastache, L. A. A.; Martínez, G. A. and Castillo, M. A. 1999b. Los mejores predictores lineales e insesgados (MPLI) en los diseños uno y tres de griffing. Agrociencia. 33(3):349-359.

Mastache, L. A. A. and Martínez, G. A. 2003. Un algoritmo para el análisis, estimación y predicción en experimentos dialélicos balanceados. Rev. Fitot. Mex. 26(3):191-200.

Mbusa, H. K.; Ngugi, K.; Olubayo, F. M.; Musembi, K. B.; Muthomi, J. W. and Nzuve, F. M. 2017. The Inheritance of yield components and beta carotene content in sweet potato. J. Agric. Sci. 10(2):71-81.

Moore, K. J. and Dixon, P. M. 2014. Analysis of combined experiments revisited. Agron. J. 107(2):763-771.

Muhinyuza, J. B.; Shimelis, H.; Melis, R.; Sibiya, J. and Nzaramba, M. N. 2016. Combining ability analysis of yield and late blight [Phytophthora infestans (Mont.) de Bary] resistance of potato germplasm in rwanda. Austr. J. Crop Sci. 10(6):799-807.

Muhumuza, E.; Edema, R.; Namugga, P. and Barekye, A. 2020. Combining ability analysis of dry matter content, reducing sugars and yield of potato (Solanum tuberosum L.) genotypes in uganda. J. Sci. Agric. 4(1):01-08.

Mulato, B. J.; Peña, L. A.; Sahagún, C. J.; Villanueva, V. C. and López, R. J. J. 2020. Selfcompatibility inheritance in tomatillo (Physalis ixocarpa Brot.). Vegetable Crops Res. Bulletin. 67(1):17-24.

Mumtaz, A.; Zafar, F. and Shehzad, S. A. 2015. A review on mating designs. Nat. Sci. 13(2):98-105.

Nduwumuremyi, A.; Tongoona, P. and Habimana, S. 2013. Mating designs: helpful tool for quantitative plant breeding analysis. J. Plant Breed. Genet. 01(03):117-129.

Ngalio, S.; Shimelis, H.; Sibiya, J.; Mtunda, K. and Mashilo, J. 2019. Combining ability and heterosis of selected sweetpotato (Ipomoea batatas L.) clones for storage root yield, yield related traits and resistance to sweetpotato virus disease. Euphytica. 215(87):1-19.

Oliveira, G. H. F.; Buzinaro, R.; Revolti, L. T. M.; Giorgenon, C. H. B.; Charnai, K; Resende, D. and Moro, G. V. 2016. An accurate prediction of maize crosses using diallel analysis and best linear unbiased predictor (BLUP). Chil. J. Agric. Res. 76(3):294-299.

Peña, L. A. y Márquez, S. F. 1990. Mejoramiento genético de tomate de cascara (Physalis ixocarpa Brot.). Rev. Chapingo. 15(71-72): 84-88.

Peña, L. A.; Molina, G. J. D.; Ortíz, C. J.; Cervantes, S. T.; Márquez, S. F. y Sahagún, C. J. 1999. Heterosis intervarietal en tomate de cascara (Physalis ixocarpa Brot.). Rev. Fitotec. Mex. 22(2):199-213.

Pérez, L. D. J.; Saavedra, G. C.; Rubí, A. M.; Franco, M. J. R. P.; Gutiérrez, R. F. y González, H. A. 2020. Código de SAS para analizar un dialélico completo y heterosis. UN ambiente. Rev. Mex. Cienc. Agríc. 11(4):829-840.

Rivera-Colín, A.; Mejía-Carranza, J.; Vázquez-García, L. M.; Urbina-Sánchez, E. y RamírezGerardo, M. G. 2019. Aptitud combinatoria y heterosis en variedades de gerbera (Gerbera x hybrida). Rev. Fitotec. Mex. 42(2):155-162.

Rodríguez, F.; Alvarado, G.; Pacheco, A.; Burgueño, J. and Crossa, J. 2018. AGD-R Software (analysis of genetic design in R), versión 5.0, CIMMYT. Unidad de biometría y estadística. El Batán, estado de México, México.

Rodríguez-Pérez, G.; Zavala-García, F.; Treviño-Ramírez, J. E.; Ojeda-Zacarías, C.; MendozaElos, M.; Rodríguez-Herrera, S. A. y Cervantes-Ortiz, F. 2016. Aptitud combinatoria y heterosis entre líneas de dos tipos de maíz para grano. Interciencia. 41(1):48-54. 
Sahagún, C. J. 1990. Utilidad del análisis de varianza en el estudio de la interacción entre genotipos y ambientes. Rev. Xilonen de la Facultad de Ciencias Agrícolas (UAEMéx). 1(1):21-32.

Sahagún, C. J. 1998. Construcción y análisis de los modelos fijos, aleatorios y mixtos. Universidad Autónoma Chapingo (UACH)-Departamento de Fitotecnia. Programa nacional de investigación en olericultura. Boletín técnico núm. 2. 64 p.

Santiaguillo, H. J. F.; Cervantes, S. T. y Peña, L. A. 2004. Selección para rendimiento y calidad de fruto de cruzas planta a planta entre variedades de tomate de cascara. Rev. Fitot. Mexic. 27(1):85-91.

Sestraş A. F.; Jäntschi, L. and Bolboacă, S. D. 2018. Using the Griffing's experimental design method I, model II. Apple breeding - a case study as a proposed methodology of the statistical and genetic analysis. Genetika. 50(1):107-120.

Singh, D. 1973. Diallel analysis for combining ability over several environments- i. Indian J. Gen. Plant Breed. 33(3):469-481.

Singh, D. 1973. Diallel cross analysis for combining ability over several environments- ii. Indian J. Gen. Plant Breed. 33(3):469-481.

Shattuck, V. I.; Christie, B. and Corso, C. 1993. Principles of griffing's combining ability analysis. Genetica. 90(1):73-79.

Sprague, G. F. and Tatum, L. A. 1942. General versus specific combining ability in single crosses of corn. J. Am. Soc. Agron. 34(10):923-832.

Soriano, V. J. M. 2000. The parametric restrictions of thegriffing diallel analysis model: combining ability analysis. Gen. Mol. Biol. 23(4):877-881.

Vasconcelos, W. S.; Dos-Santos, R. C.; Vasconcelos, U. A. A.; Cavalcanti, J. J. V. and Farias, F. J. C. 2020. Estimates of genetic parameters in diallelic populations of cotton subjected to water stress. Rev. Brasileira de Engenharia Agrícola e Ambiental. 24(8):541-546.

Vesali, M. R.; Baradaran, R.; Hassanpanah, D. and Seghatolelami, M. J. 2020. Generating genetic diversity through diallel crosses of promising potato cultivars (Solanum tuberosum L.) and studying cultivar hybrids under water deficit stress. Rev. Agricultura Neotropical, Cassilândia-MS. 7(2):49-56.

Wright, A. J. 1985. Diallel design, analyses and reference populations. Heredity. 54(3):307-311.

Yaw, O. E.; Mohammed, H.; Manigben, K. A.; Adjebeng-Danquah, J.; Kusi, F.; Karikari, B. anf Kofi, S. E. 2020. Diallel analysis and heritability of grain yield, yield components, and maturity traits in cowpea (Vigna inguiculata (L) Walp). The Sci. World J. ID 9390287. 9 p.

Zhang, Y. and Kang, M. S. 1977. DIALLEL-SAS: A SAS Program for griffing's diallel analyses. Agron. J. 89(2):176-182.

Zhang, Y.; Kang, M. S. and Lamkey, K. R. 2005. DIALLEL-SAS05: A Comprehensive program for griffing's and gardner-eberhart analyses. Agron. J. 97(4):1097-1106. 